\title{
The Use of Personal Pronouns: A Comparison between Iranian and Malaysian Dyads
}

\author{
Seyed Yasin Yazdi-Amirkhiz \\ Institute of Research Management and Monitoring \\ University of Malaya (UM), Malaysia
}

Tel: +60173059737 E-mail: yasin_yazdi@um.edu.my

Kamariah Abu Bakar

Institute for Mathematical Research, Universiti Putra Malaysia

Karim Hajhashemi

School of Education,

James Cook University, Australia

Received: 05-10-2013

doi:10.7575/aiac.ijalel.v.3n.1p.245
Accepted: 07-12-2013

Published: 01-01-2014

URL: http://dx.doi.org/10.7575/aiac.ijalel.v.3n.1p.245

\begin{abstract}
The present paper is part of a larger study which comparatively examined the collaborative discourse of two Iranian and two Malaysian dyads. The members of the dyads were all female and of the same English language proficiency. Core findings of the study on the typology and the frequency of the pronouns used by the participants in the course of eleven sessions of collaborative writing are reported. The content analysis of their pair talk for pronouns indicated that Iranian participants tended to use "I" and "you" considerably more than their Malaysian counterparts, whereas Malaysian participants were found to have a stronger tendency to use "we" more often. The findings are discussed with regard to the macro-cultural dichotomy of world cultures (collectivist/ individualist).
\end{abstract}

Keywords: Pronouns, collaborative writing, culture, collectivist, individualist

\section{Introduction}

The interlocutors' tendency to use certain personal pronouns has been linked to their cultural affiliation (e.g., Chen, Hsu \& Caropreso, 2006). According to Triandis (1993), "the most important cognitions of individualists use sentences that include I, me and mine; of collectivists, sentences that include us, we, and ours..." (p. 156). Li and Wang (2004) similarly ascribe the tendency towards using a particular pronoun to the cultural orientation of the discourse producers.

A number of research studies do exist in the literature, which have investigated the relationship between the use of pronouns and the cultural affiliation of the interlocutors. For example, Na and Choi's (2009) study demonstrated that the use of pronouns could reveal important characteristics of culture; the study showed that an individualistic or a collectivist orientation affects ones' use of first person singular and plural pronouns. According to the researchers, their study could establish "a truly bidirectional relationship between cultural orientation and first person pronouns [i.e., first person singular and first person plural]” (p. 1498). In a similar way, Chen, Hsu and Caropreso (2006) who crossculturally scrutinized five Taiwanese and ten Americans' online collaboration found out that Taiwanese students did use the words "we" "our" "us" in their language more often than American students who tended to use "I" "my" and "me" more frequently. The researchers attributed the findings to the influence of collectivism in Chinese culture and individualism in American culture.

Individualism vs. collectivism has been one of the salient benchmarks for the categorization of the cultures around the world. The orientation of people in different cultures towards either collectivism or individualism is typically studied in connection with the notion of "power distance Index" (PDI), that is, people's attitudes and behaviors to power and authority. Hofstede (1986) defines power distance as "the extent to which the less powerful persons in a society accept inequality in power and consider it as normal" (p.307) or it could be simply defined as the degree of accepting the other's authority. People who are low on the dimension of power distance prefer the reduction in the differences in power and authority between members of society. However, people who are high on the dimension easily tolerate the dominant inequalities and bow to them as unavoidable facts of life. His (i.e., Hofstedeh's) study showed that most cultures which rate high in collectivism also rate high in power distance, and the other way round. It is interesting to know that Malaysia with the power distance index of 104 ranks first and Iran ranks twenty-ninth with the power distance index of 58 among fifty countries and three regions. 
Now, with reference to the findings of the above studies on the use of pronouns by cultural learners on the one hand, and the connection between the PDI rank and collectivist/individualist orientation and its relevance to the use of personal pronouns on the other hand, one might wonder what the usage of pronouns would be like by Iranians and Malaysians. Thus, the main objective of the present study is to find out and describe the patterns of pronoun use, if there be any, by both Iranian and Malaysian participants of the study in connection with their standing in Hofstede's PDI scale.

\section{Method}

\subsection{Participants}

The study included 4 female Malaysian and 4 female Iranian students at a private university in Kuala Lumpur. The eight participants, who were homogeneous in terms of English proficiency, were divided into 4 dyads: 2 Malaysian dyads and 2 Iranian dyads. The Iranian dyads were named dyad A and dyad B, and the Malaysian dyads were named dyads $\mathrm{C}$ and $\mathrm{D}$.

\subsection{Data Collection Procedure}

In order to identify the patterns of using the personal pronouns by the participants, it was necessary to scrutinize the verbal interactions between the peers in the four dyads of the study across time. For this purpose, the four dyads were provided with the same graphic prompts (IELTS AM task 1) and were asked to collaboratively perform the tasks within the same time limits. The participants performed fifteen collaborative writing tasks and their verbal interactions (collaborative dialogues) taking place between the peers in each dyad were audio-recorded for the later analysis.

\subsection{Data Analysis}

The pair talk data from eleven collaborative sessions (out of fifteen sessions) was transcribed for each of the dyads. The reason behind having an equal number of collaborative session for all the dyads was to accurately quantify and reflect the pronouns used by the participants within the equal number of sessions.

The specified transcribed data were examined, the types of pronouns were identified and the frequency of them was calculated for each dyad. Some general notions and principles which were used for the analysis of pronouns are mentioned below.

For the purpose of accuracy, the performance type slips such as "... you see, if we... if we look at Laotians..." [Dyad C, Task C, L 105] were considered one pronoun. Also, the use of "you" in expressions such as "you know" were not counted as a pronoun, in that in such cases the pronoun "you" is not used to address someone, but rather the expression is used as an appeal to a shared knowledge between the interlocutors (Schiffrin, 1994).

According to Donato (1988), the way the pronouns are used, the way they are distributed and what follows the pronouns are very significant in the study and analysis of pronoun use. For instance, first person singular pronouns could be an indicator of a non-collaborative tendency on the part of a discourse producer when it is used as a kind of distancing device, an element which is meant to accentuate the individuality and individual stance ("I think"/I want/I disagree") of the discourse producer.

1. Negar: ...but I say they were... [Dyad B, task BS, L 311]

In some cases using first person singular pronouns reflects a participant's intention to control the task which is a noncollaborative orientation as well. Examples:

Niloofar: ...just employment...but I'm talking about the... ok... cross out this...

[Dyad B, Task A, L 140]

Using first person singular is not always corresponding to non-collaborative orientations, but rather collaborative tendencies. In some cases first person singular pronoun indicates a participant's agreement with the other participant's line of thought and reasoning, or sometimes reflects the fact that the person's perspective has been changed in response to the influence of his/her partner's perspective.

Teng: I think so...Maybe we can just start with foreigners...

[Dyad C, Task C, L 20]

Teng : Oh, I know what you mean now. As in the range of 10 to 2 , it is the..

[Dyad C, Task B, L 212]

Teng: Yeah, correct. So, I have got an idea from what you have said.

[Dyad C, Task B, L 149]

First person singular pronoun is sometimes used in requests or is followed by "we". In such cases using it reflects invitations to work together which is a collaborative orientation.

Gin: I think we should state the cause why the rate for UK dropped suddenly

[Dyad D, Task A, L 92]

Similarly, second person pronouns may have both collaborative and non-collaborative functions. Making use of authoritative directive discourse could be a clear indication of non-collaborative tendency. When second person pronouns are used to issue directives (examples 1-2 below) or to emphasize the other participant's individual contribution (example 3), it will have a non-collaborative orientation.

1. Niloofar ...just stop talking about exact time... 
2. Negar: ...there is a balance...

Niloofar: you wanted to say ...there is a balance... say your sentence...

[Dyad B, Task B, L 278-279]

3. You didn't finish your sentence

[Dyad B, Task A, L 77]

However, if second person pronouns are used to invite contributions, they would have collaborative orientation.

Teng: ...or right now you want to separate already?

[Dyad C, Task C, L 67]

\section{Findings}

Scrutiny of the transcribed pair talk data from the four dyads revealed consistent findings with regard to the usage of personal pronouns by the cultural dyads. The type of pronouns and the frequency of them were calculated for each dyad. The findings about the pronouns for each dyad are presented in Table 1 below.

Table 1. Type of Pronouns Used as a Percentage of Total Pronouns

\begin{tabular}{cccc}
\hline & I & YOU & WE \\
\hline Dyad A & 22 & 14 & 64 \\
Dyad B & 28 & 16 & 56 \\
Dyad C & 16 & 7 & 77 \\
Dyad D & 13 & 4 & 83 \\
\hline
\end{tabular}

As the Table 1 shows, there was a considerable discrepancy between Iranian dyads (A \& B) and Malaysian dyads (C \& D) in terms of quantitative distribution of pronouns. Whereas Malaysian dyads tended to use the first person plural pronoun "we" more often than their Iranian counterparts in their pair talks, the Iranian dyads used first person singular and second person pronouns more than Malaysian participants. The proportion of using "we" to the total pronouns was $64 \%$ and $56 \%$ for dyads $\mathrm{A}$ and $\mathrm{B}$ and $77 \%$ and $83 \%$ for dyads $\mathrm{C}$ and $\mathrm{D}$, respectively.

As for first person singular and second person pronouns, the number of cases that Malaysian dyads used "I" and "you" was smaller compared to the Iranian dyads. Whereas first person singular pronouns accounted for $16 \%$ and $13 \%$ of the total pronouns used by dyads C and D, respectively, Iranian dyads used a considerably higher percentage of first person pronouns in their collaborative discourse: $22 \%$ for dyad A and $28 \%$ for dyad B. As far as second person pronoun was concerned, the proportion was $14 \%$ and $16 \%$ for Iranian dyads and $7 \%$ and $4 \%$ for Malaysian dyads.

The point to be made here is that although quantitative analysis of pronoun distribution and frequency (represented by Table 1) brought some intra-group dynamics and developments to light, a qualitative and closer analysis of the context in which the pronouns were used could even reveal certain aspects of language use by the dyads which numerical values (quantitative analyses) normally fall short of accounting for. A finer analysis indicated that Iranians used first person singular mainly for articulating their personal positions and individual stances: 1. "I can say the diagram below..." [Dyad A, Task A, L 15]; 2. "I will explain that..." [Dyad A, Task B, L 49]; 3. "I know that" [Dyad A, Task A, L 15$] 4$. “...but I am talking about the..." [Dyad B, Task B, L 140]. 5. "I think this was better" [Dyad B, Task B, L 308$]$; 6. “...but I say they were..." [Dyad B, Task B, L 311]. However, Malaysian learners tended to use first person singular to express agreement and to consent to what was said by their interlocutors (e.g., 1. "I think so" [Dyad D, Task C, L 21]; and in many of the cases they tended to use first person singular followed by first person plural "we" to denote what ought to be performed collectively. 1. "I think we should compare..." [Dyad C, Task C, L 7]; 2. "I guess we can see the..." [Dyad C, Task B, L 15]; 3. "I think we need to insist..." [Dyad D, Task B, L 21]; 4. "I think we should state the cause..." [Dyad D, Task B, L 92].

By the same token, a qualitative analysis of the use of the second person pronouns revealed that in the case of Malaysian dyads the pronoun was majorly used for suggesting the agreement to what was said by the interlocutor; For example, 1.“As you say..." [Dyad C, Task B, L 37]; 2. "Oh, I know what you mean now. As in the range of 10 to 2, it is the..." [Dyad C, Task B, L 212]; 3. "Yeah, correct. So, I have got an idea from what you have said." [Dyad C, Task B, L: 149]. However, In the case of Iranians there were more of the instances in which the second person pronouns were used in a non-collaborative fashion; they were used for issuing directives (e.g., "no, no...look... compare yellow and grey parts" [Dyad B, L 74], or to emphasize the peer's individual contribution (e.g., "You cannot mention anything about the...”[Dyad B, Task B, L 139]; "You didn't finish your sentence” [Dyad B, Task A, L 77].

\section{Discussion and Conclusion}

Examining the collaborative discourse of Iranian and Malaysian dyads showed a discrepancy in the frequency of the pronouns used. The Iranian dyads tended to use first person singular and second person pronouns considerably more often than their Malaysian counterparts, whereas Malaysian participants were found to have a stronger tendency to use first person plural pronouns more. The predominant use of first-person and second person singular pronouns in the discourse of Iranians could be an indication of their stronger individualistic tendencies in comparison with their Malaysian counterparts. According to Donato (1988), the use of personal pronouns by group members could indicate 
the orientation of group members to the group functioning. Villamil and Guerrero (1996) have also noted that the use of first person singular and plural pronouns could distinguish collaborative from non-collaborative dyads. In other words, the more first person singular pronouns are used in the collaborative discourse of the peers, the higher degree of individualistic tendencies is expected to prevail in the group and vice versa.

The findings do also go in parallel with the predictive capacity of Hofstede's power distance index, according to which the Iranians with their ranking of twenty-nine on PDI scale are expected to display stronger individualistic tendencies compared to their Malaysian counterparts with their first rank of PDI among fifty countries and three regions. Along the same lines, Triandis (1993) contends that "the most important cognitions of individualists use sentences that include $I$, me and mine; of collectivists, sentences that include $u s$, we, and ours..." (p. 156). Other researchers whose studies have found discrepant patterns of using pronouns among cultural groups have also primarily attributed the discrepancy to the individualist/collectivist dichotomy of cultures (Chen, Hsu \& Caropreso, 2006; Li \& Wang, 2004; Na \& Choi, 2009). Thus, based on the findings obtained from this study, it could be concluded that the cultural affiliation might function as one of the influential parameters in the choice of personal pronouns.

\section{Limitations of Study}

The smallness of the size of the population in the present study precludes the generalizeability of its findings to other contexts and populations. Given the nature of the study, the small sample size was inevitable. In support of such a condition for a study, Lynch (2007) recognizes the diversity and locality of contexts, and accordingly emphasizes the importance of 'particularizing' research to one's own learning and teaching context. Moreover, since the participants of this study were all female and gender is associated with collectivist and individualist tendencies (Triandis, 1993), the findings are only applicable to females. According to Triandis

"...men are more individualistic than women. Age is slightly related to collectivism."

\section{References}

Chen, S. J., Hsu, C., \& Caropreso, E. J. (2006). Cross-cultural collaborative online learning: When the west meets the east. International Journal of Technology in Teaching and Learning, 2(1), 17-35.

Donato, R. (1988). Beyond group: A psycholinguistic rationale for collective activity in second-language learning. Unpublished doctoral dissertation. University of Delaware.

Hofstede, G. (1986). Cultural differences in teaching and learning. International Journal of Intercultural Relations, 10(3), 301-320.

Li, X., \& Wang, H. (2004). Cultural influence and beyond: Investigating L2 writing. In K. Kaur \& M. E. Vethamani (Eds.), Second language writing (pp. 93-117). Petaling Jaya: Sasbadi Sdn. Bhd.

Lynch, T. (2007). Learning from the transcripts of an oral communication task. ELT Journal, 61(4), 311-320.

Na, J., \& Choi, I. (2009). Culture and first-person pronouns. Personality and Social Psychology Bulletin, 35(11), 14921499.

Schiffrin, D. (1994). Approaches to discourse (Vol. 8): Wiley-Blackwell.

Triandis, H. C. (1993). Collectivism and individualism as cultural syndromes. Cross-Cultural Research, 27(3-4), 155.

Villamil, O. S., \& Guerrero, M. (2006). Sociocultural theory: A framework for understanding the social-cognitive dimensions of peer feedback. Feedback in second language writing: Contexts and issues, 23-42. 